\title{
Fatigue in Cancer Patients in Palliative Care-A Review on Pharmacological Interventions
}

\author{
Caritha Klasson ${ }^{1,2}$, Maria Helde Frankling ${ }^{1,2} \mathbb{(}$, Carina Lundh Hagelin ${ }^{3,4}$ (i) and Linda Björkhem-Bergman ${ }^{1,2, *}$ \\ 1 Department of Neurobiology, Care Sciences and Society (NVS), Division of Clinical Geriatrics, \\ Karolinska Institutet, Blickagången 16, Neo floor 7, SE-141 83 Huddinge, Sweden; \\ caritha.klasson@ki.se (C.K.); maria.helde.frankling@ki.se (M.H.F.) \\ 2 Palliative Medicine, Stockholms Sjukhem Foundation, Mariebergsgatan 22, SE-112 19 Stockholm, Sweden \\ 3 Department of Health Care Sciences, Ersta Sköndal Bräcke University College, Palliative Research Centre, \\ P.O. Box 11189, SE-100 61 Stockholm, Sweden; carina.lundh-hagelin@esh.se \\ 4 Department of Neurobiology, Care Sciences and Society (NVS), Division of Nursing, Karolinska Institutet, \\ Alfred Nobels Alle 23, SE-141 83 Huddinge, Sweden \\ * Correspondence: linda.bjorkhem-bergman@ki.se; Tel.: +46-(0)-733-168462
}

\section{check for} updates

Citation: Klasson, C.; Helde Frankling, M.; Lundh Hagelin, C.; Björkhem-Bergman, L. Fatigue in Cancer Patients in Palliative Care-A Review on Pharmacological Interventions. Cancers 2021, 13, 985. https://doi.org/10.3390/cancers 13050985

Academic Editors:

Mari Lloyd-Williams and

Sebastiano Mercadante

Received: 12 January 2021

Accepted: 22 February 2021

Published: 26 February 2021

Publisher's Note: MDPI stays neutral with regard to jurisdictional claims in published maps and institutional affiliations.

Copyright: (c) 2021 by the authors. Licensee MDPI, Basel, Switzerland. This article is an open access article distributed under the terms and conditions of the Creative Commons Attribution (CC BY) license (https:/ / creativecommons.org/licenses/by/ $4.0 /)$.
Simple Summary: Cancer related fatigue is a common and distressing symptom for patients with cancer during and after primary treatment, and also in the palliative phase of the disease trajectory. This review focuses on the pharmacological treatment of cancer related fatigue in patients with advanced or metastatic cancer. There are few high-quality studies performed in this setting, but both methylphenidate and corticosteroids might be used to relieve fatigue.

Abstract: Fatigue is one of the most distressing symptoms experienced by cancer patients. The suggested biological mechanism for cancer related fatigue (CRF) includes immune activation triggered by tumor tissue or by anticancer treatment but other mechanisms have also been proposed. Previous large meta-analysis of interventions on fatigue focuses mostly on patients early in the disease trajectory, with only one tenth of included studies performed in palliative cohorts. The aim of this narrative review is therefore to present a background on CRF with focus on the palliative setting. A summary of recent randomized, controlled trials on pharmacological interventions on CRF in palliative care is presented, including studies on psychostimulants, corticosteroids, testosterone and melatonin. Interestingly, in several of these studies there was a positive and similar effect on fatigue in both the intervention and the placebo arm-indicating an important placebo effect for any pharmacological treatment. In addition, studies on dietary supplements and on pharmacological complementary medicines are discussed. To conclude, the evidence is still weak for using pharmacological treatments on CRF in palliative care patients-although methylphenidate and corticosteroids might be considered.

Keywords: fatigue; palliative care; cancer; cancer related fatigue; clinical trials; therapeutics

\section{Introduction}

\subsection{Cancer Related Fatigue}

Fatigue is one of the most distressing symptoms experienced by patients with cancer and has a high prevalence in cancer patients [1]. Fatigue can occur before, during and after treatment and persist for a long time [2,3]. Different definitions have been used, but today the most widely used definition stems from the National Comprehensive Cancer Network, NCCN, "Cancer-Related Fatigue is a distressing, persistent, subjective sense of physical, emotional, and/or cognitive tiredness or exhaustion related to cancer or cancer treatment that is not proportional to recent activity and interferes with usual functioning" [4].

The concept of fatigue as a multidimensional symptom has been challenged by the notion that fatigue rather should be regarded as a set of multiple symptoms to be addressed 
separately [5,6]. The trajectory of fatigue may also change in accordance with development of the cancer disease, with physical but not mental fatigue reported to be more severe in advanced stage cancer patients compared to cancer survivors or healthy individuals [7-9]. Cancer related fatigue (CRF) could have a negative impact on patients physical, psychological, social and existential wellbeing, with an impaired experience of quality of life (QoL) as a result [3]. Most research regarding treatment strategies to reduce CRF has been performed in younger patients, with a bias towards women with breast cancer during or after primary treatment [10].

Various measurements are being used to assess presence, frequency and severity of symptoms in palliative care $[7,11,12]$. The presence of fatigue in patients with cancer with palliative needs is of risk of being overseen by health care personnel due to difficulties in identifying the symptom but also due to a lack of enhanced knowledge of mechanisms causing fatigue. There is a risk that patients suffering from fatigue underreport the symptom if assessment is not made or if health care personnel do not acknowledge the symptom and bring it up for discussion [13]. Patient reported outcome might differ from physician reported outcome [14], suggesting the importance of using validated assessment tools when measuring fatigue.

\subsection{Cancer Related Fatigue in Patients in Palliative Care}

The World Health Organization (WHO) stresses the importance of alleviating distressing symptoms to improve QoL in patients suffering from a life shortening disease [15]. Early identification and symptom management has a key role in palliative care regarding the patients' physical, psychosocial and existential needs. The improvement of treatments of non-curable diseases can facilitate a prolonged lifetime for patients with life shortening diseases, hence the importance of an active palliative care to improve QoL [16]. The recently proposed revision of the definition of palliative care emphasizes the need for evidence-based practice [17].

Continuous improvement of oncological treatment strategies has steadily improved survival times for patients with cancer in the palliative phase. During these longer disease trajectories, fatigue is a clinical problem for many individuals. Aggregated data from studies on cancer patients with fatigue as a primary outcome show that physical activity and psychological interventions are the best method to alleviate symptoms [10]. However, increased physical activity may not always be an option in the palliative phase, and pharmacological methods must therefore be considered. Neither have psychological interventions been shown to relieve fatigue in patients with advanced stage cancer during and after primary treatment [18], in contrast to patients earlier in their disease trajectory [10]. There is a lack of studies on both physical activity and psychological interventions on patients in the late palliative phase of the cancer disease.

In a previous study on fatigue in patients admitted to palliative care $(n=228)$ it was shown that fatigue increased closer to death [8]. However, fatigue was more distressing and associated with impaired QoL in patients with 2-6 months left in life while the association between fatigue and QoL disappeared during the last days and weeks of life [8].

\subsection{Previous Reviews and Meta-Analyses of Cancer Related Fatigue, Aim of the Present Review}

Since data on interventions regarding CRF have been gathered in a variety of different settings using different assessment tools, there has been a large interest in aggregating data in reviews and meta-analyses of interventions, to be able to draw firmer conclusions regarding best practice. In systematic reviews targeting all aspects of CRF, no subgroup analysis of palliative cohorts has been performed. Reviews focused on data on palliative patients have also included patients with non-cancer diseases $[10,19,20]$, were published some years ago and do therefore not include results from recently published randomized controlled trials (RCTs) [1,21], or focus solely on one type of pharmacological treatment [22]. No review has yet evaluated data specifically on pharmacological treatment of fatigue in palliative cancer patients. The scope of this narrative review is therefore to present a 
background on CRF with focus on the palliative setting, followed by a thorough review of the literature on presently used pharmacological agents and a discussion of best practice and need for further research. An overview of published reviews in the field from 2014 to 2020 are presented in Table 1.

Table 1. Reviews on cancer related fatigue.

\begin{tabular}{|c|c|c|c|c|c|}
\hline Review & Mücke [19] & Yennurajalingam [1] & Qu [21] & Mustian [10] & Tomlinson [20] \\
\hline Time, until & April 2014 & June 2014 & July 2014 & May 2016 & May 2017 \\
\hline Type & $\begin{array}{c}\text { Systematic review, } \\
\text { MA }\end{array}$ & Narrative review & $\begin{array}{c}\text { Systematic review, } \\
\text { MA }\end{array}$ & $\begin{array}{c}\text { Systematic review, } \\
\text { MA }\end{array}$ & $\begin{array}{c}\text { Systematic review, } \\
\text { MA }\end{array}$ \\
\hline Inclusion criteria & $\begin{array}{l}\text { RCTs of adults in } \\
\text { palliative care } \\
\text { (cancer, } \\
\text { non-cancer) }\end{array}$ & $\begin{array}{l}\text { RCTs of adults in } \\
\text { palliative care } \\
\text { (cancer, non-cancer) }\end{array}$ & $\begin{array}{l}\text { RCTs of adults with } \\
\text { cancer }\end{array}$ & $\begin{array}{l}\text { RCTs of adults with } \\
\text { cancer }\end{array}$ & $\begin{array}{c}\text { RCTs and } \\
\text { quasi-RCTs, patients } \\
\text { with cancer or } \\
\text { recipients of HSCT }\end{array}$ \\
\hline Cancer stage & $\begin{array}{l}\text { Advanced } \\
\text { /metastatic }\end{array}$ & All stages & All stages & All stages & All stages \\
\hline Objective & $\begin{array}{l}\text { To compare effect of } \\
\text { pharmacological } \\
\text { treatment of fatigue } \\
\text { to control } \\
\text { interventions }\end{array}$ & $\begin{array}{c}\text { To review } \\
\text { pharmacological } \\
\text { interventions for } \\
\text { CRF. }\end{array}$ & $\begin{array}{l}\text { To examine the effect } \\
\text { and safety of } \\
\text { Methylphenidate and } \\
\text { Modafinil in } \\
\text { treatment of CRF }\end{array}$ & $\begin{array}{c}\text { To compare effect of } \\
\text { exercise, } \\
\text { psychological, a } \\
\text { combination thereof, } \\
\text { or } \\
\text { pharmacological } \\
\text { interventions }\end{array}$ & $\begin{array}{c}\text { To compare effect of } \\
\text { pharmacological } \\
\text { treatment of fatigue } \\
\text { to control } \\
\text { interventions }\end{array}$ \\
\hline Outcome & $\begin{array}{c}\text { Fatigue severity + } \\
\text { change }\end{array}$ & $\begin{array}{l}\text { Fatigue severity } \\
\text { (CRF) }\end{array}$ & $\begin{array}{l}\text { Fatigue severity } \\
\text { (CRF) }\end{array}$ & $\begin{array}{l}\text { Fatigue severity } \\
\text { (CRF) }\end{array}$ & $\begin{array}{c}\text { Fatigue severity + } \\
\text { change }\end{array}$ \\
\hline $\begin{array}{c}\text { Included } \\
\text { studies/subjects }\end{array}$ & $45 / 4696$ & $18 / 2855$ & $10 / 1582$ & $113 / 11525$ & $117 / 19819$ \\
\hline $\begin{array}{l}\text { Palliative studies } \\
\text { (cancer) }\end{array}$ & $40 \%$ & $\begin{array}{l}\text { Not reported } \\
\text { (narrative) }\end{array}$ & Not reported & $\begin{array}{l}10 \% \text { metastatic, } \\
30 \% \text { mixed }\end{array}$ & $17 \%$ \\
\hline $\begin{array}{l}\text { Conclusions } \\
\text { regarding } \\
\text { pharmacological } \\
\text { interventions }\end{array}$ & $\begin{array}{c}\text { Methylphenidate } \\
\text { may be } \\
\text { advantageous. Too } \\
\text { little } \\
\text { evidence for } \\
\text { corticosteroids. Not } \\
\text { enough evidence to } \\
\text { support use of } \\
\text { specific drug. }\end{array}$ & $\begin{array}{l}\text { Adheres to } \\
\text { guidelines (2014). } \\
\text { Recommends future } \\
\text { research with more } \\
\text { personalized } \\
\text { interventions. }\end{array}$ & $\begin{array}{l}\text { Methylphenidate but } \\
\text { not Modafinil } \\
\text { reduced CRF and } \\
\text { may be beneficial for } \\
\text { the treatment of CRF. }\end{array}$ & $\begin{array}{c}\text { Exercise, } \\
\text { psychological } \\
\text { intervention + their } \\
\text { combination reduces } \\
\text { CRF. As a group, } \\
\text { pharmaceutical } \\
\text { interventions are not } \\
\text { effective during and } \\
\text { after cancer } \\
\text { treatment. }\end{array}$ & $\begin{array}{c}\text { As a group, } \\
\text { stimulants were not } \\
\text { effective. } \\
\text { Methylphenidate } \\
\text { improved fatigue, } \\
\text { while Modafinil and } \\
\text { corticosteroids did } \\
\text { not. }\end{array}$ \\
\hline
\end{tabular}

Abbreviations: MA: meta-analysis, RCT: randomized controlled trial, CRF: cancer related fatigue.

\subsection{Epidemiology of Cancer Related Fatigue with Focus on Patients in Palliative Care}

Two systematic reviews with meta-analyses on the prevalence of fatigue in cancer patients (all stages) have recently been published [12,23-25], with frequency of fatigue in the pooled data reported to be 52 and $49 \%$ respectively. A comparison of these two metaanalyses is presented in Table 2. Neither study performed a subgroup analysis of cancer patients in the palliative phase. However, in the study by Ma et al., lower performance status was assessed as the most prominent risk factor for CRF [25]. Similarly, Al Maqbali et al. noted the highest frequency of CRF in patients with advanced disease [12]. In a nationwide Australian study on routinely collected data from more than 116,000 subjects, $80 \%$ of cancer patients reported fatigue during the last 60 days of life, and $50 \%$ assessed fatigue level to be moderate or severe [26]. 
Table 2. Comparison of two meta-analyses on the prevalence of cancer related fatigue.

\begin{tabular}{|c|c|c|}
\hline Study & Ma et al. [25] & Al Maqbali et al. [12] \\
\hline Inclusion/exclusion criteria & $\begin{array}{l}\text { Observational studies } \\
\text { (>50 participants) on patients with cancer. } \\
\text { Included studies reported diagnostic } \\
\text { criteria for CRF and prevalence of CRF or } \\
\text { risk factors of CRF. }\end{array}$ & $\begin{array}{c}\text { Cross sectional or baseline data from } \\
\text { cohort studies ( }>50 \text { participants), patients } \\
\text { with cancer aged }>15 \text {. Fatigue measured } \\
\text { on multi-item scales. }\end{array}$ \\
\hline $\begin{array}{l}\text { Number of screened/ } \\
\text { included articles }\end{array}$ & $2641 / 84$ & $10,516 / 129$ \\
\hline Number of subjects & $144,813(31 \%$ male $)$ & 71,568 (51\% male) \\
\hline Cancer related fatigue (\%) & $52(95 \%$ CI 48-56) & 49 (95\% CI 45-53) \\
\hline Subgroup analysis & $\begin{array}{l}\text { Risk factors for fatigue } \\
\quad(O R, 95 \% \mathrm{CI})\end{array}$ & $\begin{array}{l}\text { Frequency } \\
(\%)\end{array}$ \\
\hline \multirow[t]{6}{*}{ Strongest association } & Poor performance status $(6.58,2.60-16.67)$ & Ongoing treatment (62) \\
\hline & Insomnia $(2.83,1.22-6.57)$ & Advanced stage (61) \\
\hline & Pain $(2.64,1.20-5.80)$ & Mixed cancers in study (57) \\
\hline & $\begin{array}{l}\text { Chemoradiotherapy } \\
(2.25,1.90-2.67)\end{array}$ & \\
\hline & Depression $(2.23,1.70-2.92)$ & \\
\hline & Female sex $(2.07,1.51-2.84)$ & \\
\hline \multirow[t]{2}{*}{ Weakest association } & Neuroticism $(1.23,1.05-1.43)$ & \\
\hline & $\begin{array}{l}\text { No statistical significance: Low income, } \\
\text { comorbidities, anxiety, physical exercise }\end{array}$ & \\
\hline $\begin{array}{l}\text { Separate analysis of patients in } \\
\text { palliative phase }\end{array}$ & No & No \\
\hline
\end{tabular}

Abbreviations. CRF: cancer related fatigue, CI: confidence interval, OR: odds ratio.

\subsection{Etiology of Cancer Related Fatigue}

Fatigue in cancer patients in the palliative phase may be due to both preventable and treatable concurrent conditions, e.g., depression, infections, anemia, sleep disorders, pain, vitamin imbalance, etc. (Figure 1). These should be identified through a detailed medical history, physical examination and relevant investigations [4] and treated according to guidelines/best practice with regard to patients' wishes and functional status [24]. Additionally, side effects of active oncological treatment include fatigue, and it is important to screen patients in a palliative phase of their disease trajectory for self-assessed fatigue to optimize systemic oncological treatment. Further, fatigue is as such a self-assessed, subjective measure subject to variations in social, emotional and existential status [3,27].

The mechanistic, pathophysiologic underpinning for cancer related fatigue that remains when other factors have been treated/excluded, so called "primary fatigue" according to European Association of Palliative Care (EAPC) 2008 [2], is elusive, but several underlying mechanisms have been proposed in recent reviews [3,28,29]: peripheral immune activation and inflammatory dysfunction due to both to immune system response to tumor tissue and triggered by anticancer treatment; skeletal muscle and mitochondrial dysfunction (in conjunction with cancer cachexia) and neuronal disorders. This adheres well to the categorization of causative concepts by Bower et al. in 2014 in two categoriesinflammation and neuroendocrine alterations. 


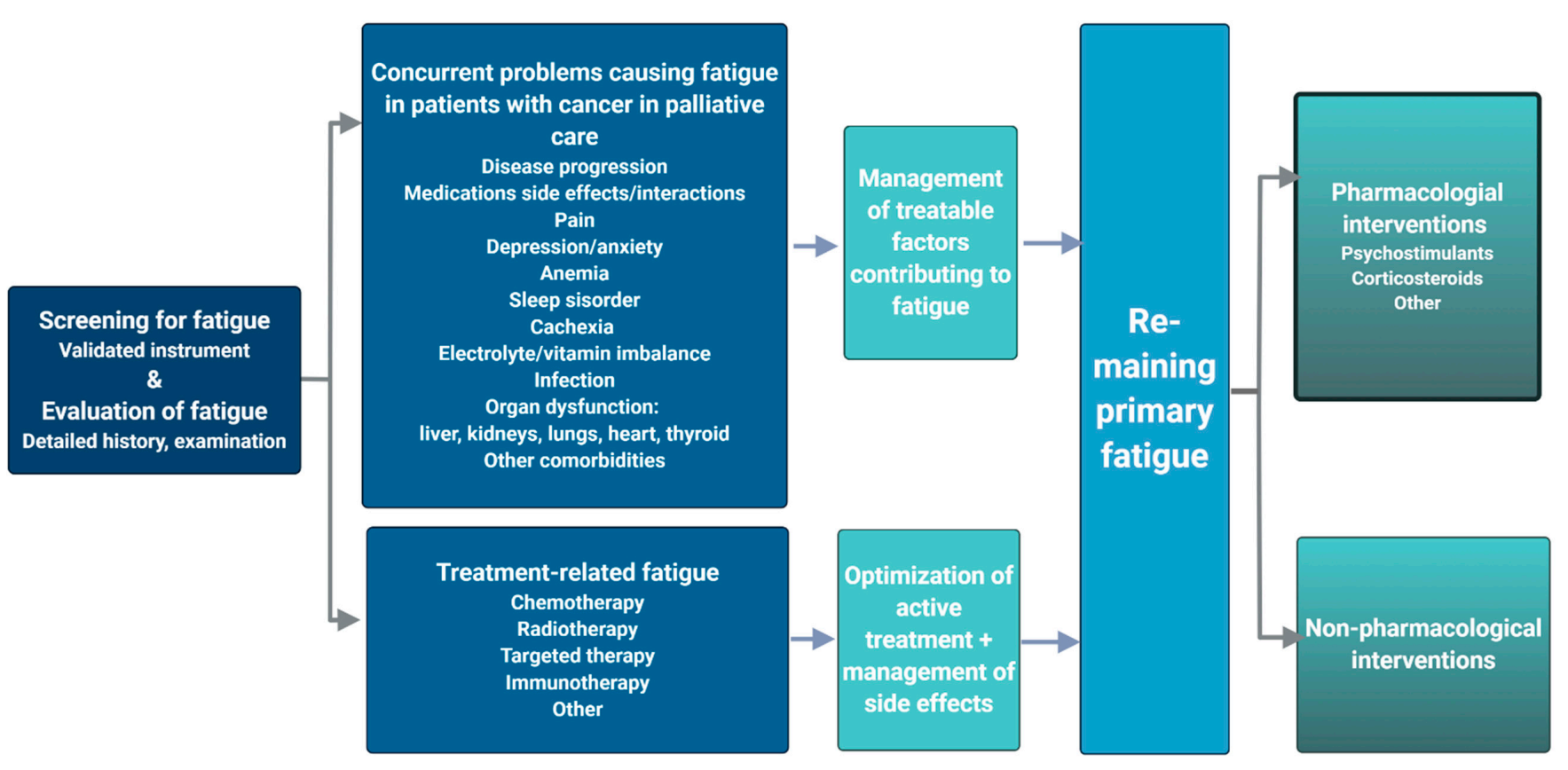

Figure 1. Etiology and management of cancer related fatigue. Made with Biorender.com. Adopted from similar figures in previous work $[4,13,23,24]$.

The etiology of fatigue may differ in the early palliative phase compared to the late phase. In the early phase, fatigue and change in fatigue often show an association with inflammation biomarkers [30]. However, in the late stage, close to death, an improvement in fatigue is often difficult to achieve and recovery of inflammatory biomarkers is seldom possible. The experience of fatigue in the very last days of life is also influenced by other symptoms, and the fact that life comes to an end. Despite the same influential etiology, the experience and meaning of fatigue can differ in End-of-Life compared to earlier palliative phases [8].

Both the European Society for Medical Oncology, ESMO and the United States National Comprehensive Cancer Network (NCCN), and other national medical organizations publish consensus-based clinical practice guidelines regarding management of cancer related fatigue with recent updates available [4,31,32]. ESMO does not differentiate between patients in different phases of their disease in their guidelines, but considers patients aged 65 or above separately, since little data on both fatigue assessment and best treatment practice is available for this group [31]. NCCN identifies three groups of cancer patients: undergoing active treatment with focus on curative treatment, post treatment patients and patients in End-of-Life, but does not specifically consider patients in an earlier palliative phase [4]. According to NCCN guidelines, both methylphenidate and shorter courses of corticosteroids can be considered in End-of-Life management of CRF. ESMO, on the other hand, could not reach a consensus on the benefits regarding methylphenidate, but advocates shorter courses of corticosteroids in patients with metastatic cancer [31]. No other pharmacological interventions are deemed beneficial in this setting by either ESMO or NCCN.

\section{Materials and Methods}

This review is conducted as a narrative review of pharmacological interventions on CRF in palliative care to present an overview of different studies being performed up until the date November 2020. Searches have been performed in Medline with the following term both as $\mathrm{MeSH}$ term and as free text: fatigue, neoplasm, therapeutics, cancer related fatigue, palliative care, randomized controlled trials and treatment outcome. The terms have been combined in different search blocks of three or more terms combined. More than 500 titles 
have been screened. In addition, the systematic reviews and meta-analysis mentioned above have been reviewed to find eligible studies $[1,10,12,19-21,25]$. The inclusion criteria for studies presented in Table 3 were randomized, controlled trials, patients $\geq 18$ years with any type of advanced cancer and/or in a palliative care setting with a pharmacological intervention with a control group and with outcome measure of effect on CRF.

\section{Results}

In Table 3 all randomized controlled trials on pharmacological interventions fulfilling the inclusion criteria are presented. In total, 17 studies were included in this review with a total of 1296 patients participating in the trials. The primary objective of the studies was improvement in fatigue, CRF and treatment induced fatigue in patients with cancer. Patient reported outcomes were measured with a variety of different assessment tools.

Table 3. Summary of randomized controlled trials on pharmacological treatments of fatigue in patients with advanced cancer.

\begin{tabular}{|c|c|c|c|c|c|c|}
\hline Study & Population & Study Design & Intervention & $\begin{array}{l}\text { Comparative } \\
\text { Intervention }\end{array}$ & $\begin{array}{c}\text { Primary } \\
\text { Outcome } \\
\text { (Assessment Tool) }\end{array}$ & Comments \\
\hline \multicolumn{7}{|c|}{ Methylphenidate (MPH) } \\
\hline $\begin{array}{l}\text { Centeno } 2020 \text { [33] } \\
\text { Spain } \\
\text { Advanced } \\
\text { cancer }\end{array}$ & $\begin{array}{l}\text { ITT }=100, \mathrm{PP}=77 \\
\text { Intervention: Mean } \\
\text { age }=66 \\
\text { Men }=52 \% \\
\text { Placebo: } \\
\text { Mean age }=68 \\
\text { Men }=53 \%\end{array}$ & $\begin{array}{l}\text { Randomized, } \\
\text { double blind, } \\
\text { placebo } \\
\text { controlled }\end{array}$ & $\begin{array}{c}\text { Methylphenidate } \\
\text { 10-25 mg/day for } 6 \\
\text { days }\end{array}$ & Placebo & $\begin{array}{l}\text { Effect on fatigue } \\
\text { after } 6 \text { days. } \\
\text { (ESAS, FACT-F). }\end{array}$ & $\begin{array}{l}\text { No significant } \\
\text { difference between } \\
\text { treatment arms } \\
\text { (ESAS } p=0.52, \\
\text { FACT-F } p=0.3 \text { ). } \\
\text { Mean improvement } \\
\text { in MPH group: } \\
\text { ESAS - 2.3 (SD 2.6), } \\
\text { FACT-F -3.4 } \\
\text { (SD 2.5) } \\
\text { Placebo group: } \\
\text { ESAS - 1.9 (SD 2.5), } \\
\text { FACT-F -2.4 } \\
\text { (SD 2.9) }\end{array}$ \\
\hline $\begin{array}{c}\text { Pedersen } 2020 \text { [34] } \\
\text { Denmark } \\
\text { Cancer, } \\
\text { palliative care. }\end{array}$ & $\begin{array}{l}\text { ITT }=38, \mathrm{PP}=28 \\
\text { Intervention: } \\
\text { Mean age: } 69 \\
\text { Men: } 29 \% \\
\text { Placebo: - }\end{array}$ & $\begin{array}{l}\text { Randomized, } \\
\text { double blind, } \\
\text { placebo } \\
\text { controlled }\end{array}$ & $\begin{array}{c}10 \text { tablets of } \\
\text { Methylphenidate } \\
10 \mathrm{mg} \text { and } 10 \text { tablets } \\
\text { placebo, } \\
\text { randomly packed }\end{array}$ & $\begin{array}{c}\text { Placebo, } \\
\text { own control. }\end{array}$ & $\begin{array}{l}\text { Effect on fatigue } \\
\text { after } 2 \text { and } 5 \mathrm{~h} . \\
\text { (VAS tiredness). }\end{array}$ & $\begin{array}{l}\text { Significant effect } \\
\text { with MPH but not } \\
\text { placebo after } 2 \mathrm{~h} \\
\text { (mean difference in } \\
\text { decrease }-12, \text { SD 20, } \\
p=0.004 \text { ) and after } \\
5 \mathrm{~h} \text { (mean difference } \\
\text { in decrease }-12, \mathrm{SD} \\
19, p=0.001 \text { ) }\end{array}$ \\
\hline $\begin{array}{c}\text { Richard } 2015 \text { [35] } \\
\text { Canada } \\
\text { Advanced } \\
\text { Prostate cancer }\end{array}$ & $\begin{array}{l}\text { ITT = 24, PP = } 23 \\
\text { Intervention: } \\
\text { Median age }=63 \\
\text { Men = 100\% } \\
\text { Placebo: } \\
\text { Median age = } 74 \\
\text { Men }=100 \%\end{array}$ & $\begin{array}{l}\text { Randomized, } \\
\text { double blind, } \\
\text { placebo } \\
\text { controlled }\end{array}$ & $\begin{array}{l}\text { Methylphenidate } \\
\text { 5-10 mg/day for } \\
12 \text { weeks }\end{array}$ & Placebo & $\begin{array}{l}\text { Effect on fatigue } \\
\text { after } 10 \text { weeks } \\
\text { (FACT-F). }\end{array}$ & $\begin{array}{l}\text { After } 10 \text { weeks } \\
\text { mean difference in } \\
\text { change from } \\
\text { baseline was } 5.6 \\
\text { points in favor of } \\
\text { intervention }(95 \% \\
\text { CI } 1.0-10.3) \text {, } \\
p=0.022 .\end{array}$ \\
\hline $\begin{array}{c}\text { Mitchell } 2015[36] \\
\text { Australia } \\
\text { Advanced } \\
\text { cancer }\end{array}$ & $\begin{array}{l}\text { ITT }=43, \mathrm{PP}=24 \\
\text { Intervention: } \\
\text { Median age }=71 \\
\text { Men = 52\% } \\
\text { Placebo: - }\end{array}$ & $\begin{array}{c}\text { Randomized, } \\
\text { N-of-1, double } \\
\text { blind, placebo } \\
\text { controlled crossover, } \\
\text { multicycle } \\
\text { design. }\end{array}$ & $\begin{array}{c}\text { Methylphenidate } \\
5 \mathrm{mg} \times 2 \text { for } 3 \text { days, } \\
\text { placebo for } 3 \text { days, } \\
\text { methylphenidate } \\
5 \mathrm{mg} \times 2 \text { for } 3 \text { days } \\
3 \text { cycles. }\end{array}$ & $\begin{array}{l}\text { Placebo for } 3 \text { days, } \\
\text { Methylphenidate } \\
\text { for } 3 \text { days, } \\
\text { placebo for } 3 \text { days. } \\
3 \text { cycles. }\end{array}$ & $\begin{array}{l}\text { Effect on fatigue as } \\
\text { individual } \\
\text { comparison }+ \\
\text { population } \\
\text { estimate } \\
\text { (FACIT-F). }\end{array}$ & $\begin{array}{l}\text { No difference was } \\
\text { detected between } \\
\text { groups } \\
\text { characterized as } \\
\text { responders and } \\
\text { non-responders } \\
\text { after } 84 \text { completed } \\
\text { cycles, mean } \\
\text { difference } 3.2 \text { ( } 95 \% \\
\text { credible interval } \\
-2.0,9.0) .7 \text { patients } \\
\text { had clinically } \\
\text { significant positive } \\
\text { effect of MPH. }\end{array}$ \\
\hline $\begin{array}{c}\text { Escalante 2014 [37] } \\
\text { USA } \\
\text { Breast cancer } \\
\text { (local/ } \\
\text { metastatic) }\end{array}$ & $\begin{array}{l}\text { ITT }=42, \mathrm{PP}=33 \\
\text { Intervention: } \\
\text { Mean age = } 57 \\
\text { Men =0\% } \\
\text { Placebo: - }\end{array}$ & $\begin{array}{l}\text { Randomized, } \\
\text { placebo } \\
\text { controlled, } \\
\text { crossover }\end{array}$ & $\begin{array}{c}\text { Methylphenidate } 18 \\
\text { mg/day for } 14 \text { days } \\
\text { + placebo for } \\
14 \text { days. }\end{array}$ & $\begin{array}{l}\text { Placebo for } 14 \text { days } \\
\text { + methylphenidate } \\
18 \mathrm{mg} / \text { day for } \\
14 \text { days. }\end{array}$ & $\begin{array}{c}\text { Effect on fatigue } \\
\text { assessed as } \\
\text { improvement of } \\
\text { worst level of } \\
\text { fatigue after } 14 \text { days. } \\
\text { (BFI) }\end{array}$ & $\begin{array}{l}\text { No significant } \\
\text { difference between } \\
\text { treatment groups } \\
(p=0.54) \text { regarding } \\
\text { worst level of } \\
\text { fatigue } \\
\text { after } 14 \text { days of } \\
\text { treatment. }\end{array}$ \\
\hline
\end{tabular}


Table 3. Cont.

\begin{tabular}{|c|c|c|c|c|c|c|}
\hline Study & Population & Study Design & Intervention & $\begin{array}{l}\text { Comparative } \\
\text { Intervention }\end{array}$ & $\begin{array}{c}\text { Primary } \\
\text { Outcome } \\
\text { (Assessment Tool) }\end{array}$ & Comments \\
\hline $\begin{array}{c}\text { Bruera } 2013 \text { [38] } \\
\text { USA } \\
\text { Advanced } \\
\text { cancer }\end{array}$ & $\begin{array}{l}\mathrm{ITT}=190, \mathrm{PP}=140 \\
\text { Intervention: } \\
\text { Mean age = } 58 \\
\text { Men = 33\% } \\
\text { Placebo: - }\end{array}$ & $\begin{array}{l}\text { Randomized, } \\
\text { 4-arm, } \\
\text { placebo } \\
\text { controlled }\end{array}$ & $\begin{array}{c}\text { Methylphenidate } \\
5-20 \mathrm{mg} / \text { day }+ \\
\text { nurse telephone } \\
\text { intervention OR } \\
\text { control telephone } \\
\text { intervention for } \\
15 \text { days }\end{array}$ & $\begin{array}{l}\text { Placebo + nurse } \\
\text { telephone } \\
\text { intervention OR } \\
\text { control telephone } \\
\text { intervention for } \\
15 \text { days }\end{array}$ & $\begin{array}{l}\text { Effect on fatigue } \\
\text { after } 15 \text { days } \\
\text { (FACIT-F) }\end{array}$ & $\begin{array}{l}\text { All groups showed } \\
\text { significant effect in } \\
\text { improved fatigue } \\
\text { on day } 15 \text {. MPH } \\
\text { was not superior to } \\
\text { placebo from } \\
\text { baseline to end of } \\
\text { trial (5.5 vs. } 6.0 \text {, } \\
p=0.69) \text {. }\end{array}$ \\
\hline $\begin{array}{c}\text { Roth } 2010 \text { [39] } \\
\text { USA } \\
\text { Advanced } \\
\text { prostate cancer }\end{array}$ & $\begin{array}{l}\text { ITT }=32, \mathrm{PP}=23 \\
\text { Intervention: } \\
\text { Mean age }=68 \\
\text { Men }=100 \% \\
\text { Placebo: } \\
\text { Mean age }=71 \\
\text { Men }=100 \%\end{array}$ & $\begin{array}{l}\text { Randomized, } \\
\text { double blind, } \\
\text { placebo } \\
\text { controlled }\end{array}$ & $\begin{array}{l}\text { Methylphenidate } \\
\text { 5-30 mg for } 6 \text { weeks. } \\
\text { Individual } \\
\text { titration of dose } \\
\text { after day } 3 .\end{array}$ & Placebo & $\begin{array}{l}\text { Effect on fatigue } \\
\text { after } 6 \text { weeks. } \\
\text { (BFI). }\end{array}$ & $\begin{array}{l}\text { Significant effect of } \\
\text { both MPH and } \\
\text { placebo } \\
\text { (improvement in } \\
\text { BFI total score } 3.63 \text {, } \\
p=0.01 \text { and } 2.58, \\
p=0.02 \text { ), } \\
\text { comparison } \\
\text { between groups not } \\
\text { shown. } \\
\text { Methylphenidate } \\
\text { reduced BFI } \\
\text { severity score more } \\
\text { than placebo } \\
\text { ( } p=0.03 \text { ). RR for } \\
\text { fatigue } \\
\text { improvement in } \\
\text { MPH group was } \\
\text { 3.04 (CI 1.04-8.86) } \\
\text { compared to } \\
\text { placebo ( } p=0.02 \text { ) }\end{array}$ \\
\hline \multicolumn{7}{|c|}{ Modafinil } \\
\hline $\begin{array}{c}\text { Lee } 2016 \text { [40] } \\
\text { USA } \\
\text { Glioma }\end{array}$ & $\begin{array}{l}\text { ITT }=81, \mathrm{PP}=62 \\
\text { Intervention: } \\
\text { Median age }=56 \\
\text { Men }=57 \% \\
\text { Placebo: } \\
\text { Median age }=54 \\
\text { Men }=53 \%\end{array}$ & $\begin{array}{l}\text { Randomized, } \\
\text { placebo } \\
\text { controlled, } \\
\text { multicenter } \\
\text { pilot study }\end{array}$ & $\begin{array}{c}\text { Armodafinil } 150 \mathrm{mg} \\
8 \text { weeks during } \\
\text { radiotherapy. } \\
\text { Intervention start } \\
\text { within } 10 \text { days of } \\
\text { RT start. }\end{array}$ & Placebo & $\begin{array}{l}\text { Effect on fatigue } \\
\text { after } 42 \text { days } \\
\text { (FACIT-F). }\end{array}$ & $\begin{array}{l}\text { No significant } \\
\text { difference in } \\
\text { median change in } \\
\text { FACIT-F was } \\
\text { detected between } \\
\text { armodafinil, }-1 \\
\text { (range }-22 \text { to } 48 \text { ) } \\
\text { and placebo, }-3 \\
\text { (range }-38 \text { to } 22 \text { ), } \\
p=0.30 \text {. }\end{array}$ \\
\hline $\begin{array}{c}\text { Spathis } 2014 \text { [41] } \\
\text { UK } \\
\text { Advanced } \\
\text { lung cancer }\end{array}$ & $\begin{array}{l}\text { ITT }=208, \text { PP }=160 \\
\text { Intervention: } \\
\text { Median age }=68 \\
\text { Men }=49 \% \\
\text { Placebo: } \\
\text { Median age }=69 \\
\text { men }=50 \%\end{array}$ & $\begin{array}{c}\text { Randomized, } \\
\text { double blind, } \\
\text { placebo controlled }\end{array}$ & $\begin{array}{c}\text { Modafinil } \\
100 \mathrm{mg} \text { day } 1-14 \\
200 \mathrm{mg} \text { day } 15-28\end{array}$ & Placebo & $\begin{array}{c}\text { Effect on fatigue on } \\
\text { day } 28 \\
\text { (FACIT-F). }\end{array}$ & $\begin{array}{l}\text { No significant effect } \\
\text { between treatment } \\
\text { arms. Mean score } \\
\text { difference between } \\
\text { treatment arms } 0.20 \\
\text { (95\% CI; - 3.56-3.97) } \\
\text { based on mean } \\
\text { score change in } \\
\text { modafinil group } \\
5.29 \text { ( } 95 \% \text { CI } 2.57 \text { to } \\
8.02 \text { ) and placebo } \\
\text { group } 5.09 \text { ( } 95 \% \text { CI } \\
2.54 \text { to } 7.65) \text {. }\end{array}$ \\
\hline $\begin{array}{l}\text { Hovey } 2014 \text { [42] } \\
\text { Australia } \\
\text { Metastatic breast or } \\
\text { prostate cancer }\end{array}$ & $\begin{array}{l}\text { ITT }=83, \mathrm{PP}=66 \\
\text { Intervention: } \\
\text { Mean age }=66 \\
\text { Men }=78 \% \\
\text { Placebo: } \\
\text { Mean age }=68 \\
\text { Men }=78 \%\end{array}$ & $\begin{array}{l}\text { Randomized 2:1, } \\
\text { double blind, } \\
\text { placebo controlled, } \\
\text { multicenter study }\end{array}$ & $\begin{array}{c}\text { Day 0 + 21: } \\
\text { Chemotherapy } \\
\text { Day 3-17: } \\
\text { Modafinil } 200 \mathrm{mg} \text {. } \\
\text { Min 2, max } 4 \\
\text { cycles }\end{array}$ & Placebo & $\begin{array}{c}\text { Effect on } \\
\text { chemotherapy- } \\
\text { induced fatigue } \\
\text { (cumlative } \\
\text { MDASI AUC } \\
\text { during first } 7 \text { days } \\
\text { of TP } 1 \\
\text { and 2) }\end{array}$ & $\begin{array}{l}\text { No significant effect } \\
\text { between treatment } \\
\text { arms } \\
\text { (MDASI AUC } 3-10 \\
35.9 \text { vs } 39.6,95 \% \text { CI } \\
-8.9 \text { to } 1.4, p=0.15 \text { ). }\end{array}$ \\
\hline \multicolumn{7}{|c|}{ Dexamphetamine } \\
\hline $\begin{array}{c}\text { Auret } 2009 \text { [43] } \\
\text { Australia } \\
\text { Advanced } \\
\text { cancer }\end{array}$ & $\begin{array}{l}\text { ITT }=50, \mathrm{PP}=39 \\
\text { Intervention: } \\
\text { Mean age }=73 \\
\text { Men }=64 \% \\
\text { Placebo: } \\
\text { Mean age }=67 \\
\text { Men }=80 \%\end{array}$ & $\begin{array}{l}\text { Randomized, } \\
\text { double blind, } \\
\text { placebo } \\
\text { controlled }\end{array}$ & $\begin{array}{c}\text { Dexamphetamine } \\
10 \mathrm{mg} \times 2 \text { for } 8 \\
\text { days. }\end{array}$ & Placebo & $\begin{array}{l}\text { Effect on fatigue } \\
\text { after } 8 \text { days } \\
\text { (BFI). }\end{array}$ & $\begin{array}{l}\text { No significant } \\
\text { difference in effect } \\
\text { between groups } \\
(p=0.414) \text { at day } 8 . \\
\text { Mean decrease in } \\
\text { BFI } 1.08 \text { in } \\
\text { intervention group } \\
\text { vs. } 0.98 \text { in placebo } \\
\text { group. }\end{array}$ \\
\hline
\end{tabular}


Table 3. Cont.

\begin{tabular}{|c|c|c|c|c|c|c|}
\hline Study & Population & Study Design & Intervention & $\begin{array}{l}\text { Comparative } \\
\text { Intervention }\end{array}$ & $\begin{array}{c}\text { Primary } \\
\text { Outcome } \\
\text { (Assessment Tool) }\end{array}$ & Comments \\
\hline \multicolumn{7}{|c|}{ Melatonin (MLT) } \\
\hline $\begin{array}{c}\text { Lund } \\
\text { Rasmussen [44] } \\
2015 \\
\text { Denmark } \\
\text { Advanced } \\
\text { cancer }\end{array}$ & $\begin{array}{l}\text { ITT }=72, \mathrm{PP}=44 \\
\text { Intervention: } \\
\text { Mean age }=65 \\
\text { Men }=29 \% \\
\text { Placebo: } \\
\text { Mean age }=62 \\
\text { Men }=34 \%\end{array}$ & $\begin{array}{l}\text { Randomized, } \\
\text { placebo controlled, } \\
\text { double blind, } \\
\text { crossover }\end{array}$ & $\begin{array}{l}\text { Melatonin } 20 \mathrm{mg} \text { for } \\
1 \text { week, wash out } 2 \\
\text { days, placebo for } 1 \\
\text { week. }\end{array}$ & $\begin{array}{c}\text { Placebo for } 1 \text { week, } \\
\text { wash out } 2 \text { days, } \\
\text { Melatonin } 20 \text { mg for } \\
1 \text { week. }\end{array}$ & $\begin{array}{l}\text { Effect on fatigue } \\
\text { during first } \\
\text { intervention with } \\
\text { MLT for one week } \\
\text { (MFI-20) }\end{array}$ & $\begin{array}{l}\text { No significant effect } \\
\text { of MLT was } \\
\text { detected. Mean } \\
\text { difference in change } \\
\text { between week with } \\
\text { intervention and } \\
\text { placebo } 2.8 \text { units } \\
\text { (SD 25.6, } p=0.47 \text { ). }\end{array}$ \\
\hline \multicolumn{7}{|c|}{ Testosterone } \\
\hline $\begin{array}{c}\text { Del Fabbro } 2013 \\
\text { [45] } \\
\text { USA } \\
\text { Advanced } \\
\text { cancer, } \\
\text { hypogonadal men }\end{array}$ & $\begin{array}{l}\mathrm{ITT}=43, \mathrm{PP}=29 \\
\text { Intervention: } \\
\text { Mean age }=57 \\
\text { Men }=100 \% \\
\text { Placebo: } \\
\text { Mean age }=63 \\
\text { Men }=100 \%\end{array}$ & $\begin{array}{c}\text { Randomized, } \\
\text { double blind, } \\
\text { placebo controlled }\end{array}$ & $\begin{array}{c}\text { Testosterone } \\
150-200 \mathrm{mg}, \\
\text { injection day 1, 15, } \\
29,43,57 .\end{array}$ & Placebo & $\begin{array}{c}\text { Effect on fatigue } \\
\text { at day } 29 . \\
\text { (FACIT-F). }\end{array}$ & $\begin{array}{l}\text { No significant } \\
\text { difference in } \\
\text { fatigue scores } \\
\text { between } \\
\text { intervention (4, SD } \\
8 \text { ) and } \\
\text { placebo (-2, SD 12), } \\
p=0.12 \text {. }\end{array}$ \\
\hline \multicolumn{7}{|c|}{ Corticosteroids } \\
\hline $\begin{array}{c}\text { Paulsen } 2014 \text { [46] } \\
\text { Denmark } \\
\text { Advanced } \\
\text { cancer }\end{array}$ & $\begin{array}{l}\text { ITT }=49, \mathrm{PP}=47 \\
\text { Intervention: } \\
\text { Mean age }=62 \\
\text { Men = 50\% } \\
\text { Placebo: } \\
\text { Mean age }=66 \\
\text { Men }=52 \%\end{array}$ & $\begin{array}{c}\text { Randomized, } \\
\text { double blind, } \\
\text { placebo controlled }\end{array}$ & $\begin{array}{l}\text { Methyl- } \\
\text { prednisolone } \\
16 \mathrm{mg} \times 2 \\
\text { for } 7 \text { days. }\end{array}$ & Placebo & $\begin{array}{c}\text { Effect on pain } \\
\text { intensity after } 7 \\
\text { days. } \\
\text { Fatigue } \\
\text { secondary } \\
\text { outcome } \\
\text { (EORTC-C30). }\end{array}$ & $\begin{array}{l}\text { Significant } \\
\text { improved }(p=0.003) \\
\text { fatigue in the } \\
\text { intervention arm } \\
(-17, \text { CI } 95 \%,-27 \\
\text { to }-6) \text { compared to } \\
\text { worsened fatigue in } \\
\text { the } \\
\text { placebo arm }(3, \mathrm{CI} \\
95 \%,-5 \text { to } 11) .\end{array}$ \\
\hline $\begin{array}{l}\text { Tanioka } 2018 \text { [14] } \\
\text { Japan } \\
\text { Metastatic } \\
\text { colorectal cancer }\end{array}$ & $\begin{array}{l}\text { ITT }=74, \mathrm{PP}=72 \\
\text { Intervention: } \\
\text { Median age }=65 \\
\text { Men }=61 \% \\
\text { Placebo: } \\
\text { Median age } \mathrm{n}=68 \\
\text { Men }=63 \%\end{array}$ & $\begin{array}{c}\text { Randomized, } \\
\text { double blind, } \\
\text { placebo controlled. }\end{array}$ & $\begin{array}{l}\text { Dexamethasone } 2 \\
\mathrm{mg} \text { for } 4 \text { weeks, } 1 \\
\text { week after end of } \\
\text { targeted therapy. }\end{array}$ & Placebo & $\begin{array}{c}\text { Effect on fatigue } \\
\text { assessed as } \\
\text { incidence of fatigue } \\
\text { (CTCAE v.4). } \\
\text { Assessment by } \\
\text { patients and } \\
\text { investigators }\end{array}$ & $\begin{array}{l}\text { Significantly less } \\
\text { fatigue grade } \\
\geq 2 \text { according to } \\
\text { patients } \\
(p=0.03) \text {, but not } \\
\text { investigators } \\
(p=0.69) .\end{array}$ \\
\hline $\begin{array}{c}\text { Eguchi } 2015 \\
\text { [47] } \\
\text { Japan } \\
\text { Cancer, } \\
\text { palliative care }\end{array}$ & $\begin{array}{l}\mathrm{ITT}=35, \mathrm{PP}=34 \\
\text { Intervention: } \\
\text { Median age }=71 \\
\text { Men }=61 \% \\
\text { Placebo: } \\
\text { Median age }=68 \\
\text { Men }=62 \%\end{array}$ & $\begin{array}{c}\text { Pilot } \\
\text { randomized, } \\
\text { multicenter, double } \\
\text { blind, placebo } \\
\text { controlled }\end{array}$ & $\begin{array}{c}\text { Methyl- } \\
\text { prednisolone } \\
32 \mathrm{mg} \text { for } 7 \text { days. }\end{array}$ & Placebo & $\begin{array}{l}\text { Effect on fatigue } \\
\text { after } 7 \text { days } \\
\text { (VAS). }\end{array}$ & $\begin{array}{l}\text { No significant } \\
\text { difference between } \\
\text { groups }(p=0.484) \text {. } \\
\text { Mean change in } \\
\text { intervention arm } \\
(-1.56, \text { SD 32.5) } \\
\text { compared to } \\
\text { placebo ( }-9.06, \text { SD } \\
27.2) .\end{array}$ \\
\hline $\begin{array}{c}\text { Yennurajalingam } \\
2013 \\
{[48]} \\
\text { USA } \\
\text { Advanced } \\
\text { cancer }\end{array}$ & $\begin{array}{l}\text { ITT }=132, \mathrm{PP}=84 \\
\text { Intervention: } \\
\text { Median age }=60 \\
\text { Men = 47\% } \\
\text { Placebo: - }\end{array}$ & $\begin{array}{l}\text { Randomized, } \\
\text { double blind, } \\
\text { placebo } \\
\text { controlled }\end{array}$ & $\begin{array}{c}\text { Dexamethasone } \\
4 \mathrm{mg} \times 2 \text { for } 14 \\
\text { days }\end{array}$ & Placebo & $\begin{array}{l}\text { Effect on fatigue } \\
\text { after } 15 \text { days } \\
\text { (FACIT-F). }\end{array}$ & $\begin{array}{l}\text { Significant } \\
\text { improved in } \\
\text { intervention group } \\
\text { compared to } \\
\text { placebo, } p=0.008 \text {. } \\
\text { Mean change from } \\
\text { baseline with } \\
\text { dexamethasone was } \\
\text { 9, (SD 10.3) and } \\
\text { with placebo } 3.1 \\
\text { (SD 9.59). }\end{array}$ \\
\hline
\end{tabular}

Abbreviations: AUC: Area under Curve, BFI: Brief Fatigue Inventory; CI: Confidence Interval; CTCAE v4: National Cancer Institute's Common Terminology Criteria for Adverse Events; EORTC-C30: European Organization for Research and Treatment of Cancer-Quality of Life Questionnaire 30; ESAS: Edmonton Symptom Assessment Scale; FACIT-F: Functional Assessment of Chronic Illness Therapy-Fatigue; FACT-F: Functional Assessment of Cancer Therapy-Fatigue; I: Intervention; ITT: Intention To Treat; MDASI: MD Anderson Symptom Inventory; MFI-20: Multidimensional Fatigue Inventory-20; mg: milligram; MLT: Melatonin; MPH: Methylphenidate; PP: Per Protocol; RR: Response Rate; RT: Radio Therapy; SD: Standard Deviation; TP. Treatment Period; VAS: Visual Analogue Scale; vs: versus.

In a separate section randomized controlled studies on dietary supplements and pharmacological complementary and alternative medicine (CAM) are presented. This section includes studies performed also in non-palliative cancer patients due to the lack of palliative care studies-in contrast to the studies presented in Table 3 where only palliative care cohorts are selected. 


\subsection{Modafinil}

Several trials of the use of psychostimulant and effect on CRF have been conducted but show conflicting or weak evidence. In one randomized study of 208 patients with advanced lung cancer receiving modafinil or placebo for 28 days showed improved fatigue in both treatment arms and no difference between placebo and intervention [41]. Additionally, in a randomized placebo-controlled study of 81 patients with glioma receiving radiotherapy, armodafinil had no significant effect on fatigue [40]. A lack of significant effect on fatigue was also presented in a placebo controlled RCT in patients $(n=81)$ with advanced prostate and breast cancer receiving $200 \mathrm{mg}$ Modafinil a day during chemotherapy [42].

\subsection{Metylphenidate and Dexamphetamine}

In contrast, a placebo controlled RCT of men with prostate cancer treated with luteinizing hormone releasing hormone (LHRH) agonist $(n=24)$ revealed a significant improvement with methylphenidate $5-10 \mathrm{mg}$ /day for 10 weeks [35]. Additionally, a RCT of patients with advanced cancer $(n=38)$ showed a significant effect of methylphenidate $10 \mathrm{mg}$ two hours after intake compared to the placebo [34]. However, a dose of $20 \mathrm{mg}$ dexamphetamine for 8 days could not establish significant effect on fatigue in a study of patients with advanced cancer $(n=50)$, even if fatigue was significantly improved on day 2 [43]. Yet another study of patients with breast cancer $(n=42)$ receiving methylphenidate $18 \mathrm{mg} /$ day for two weeks could not show a significant effect over the placebo [37]. Additionally, no significant effect was evident in a placebo controlled randomized crossover study $(n=43)$ of patients with advanced cancer receiving methylphenidate $10 \mathrm{mg}$ for 3 days [36]. In contrast, improved fatigue was detected in men with advanced prostate cancer receiving methylphenidate for 6 weeks compared to the placebo group [39]. However, the study was underpowered since the intervention group only had 10 completers [39]. A mixed 4-arm placebo-controlled intervention $(n=140)$ of methylphenidate $5-20 \mathrm{mg} /$ day and nursing telephone intervention established an improvement in fatigue on day 8 but not at the end of trial at day 15 . Nor methylphenidate or nursing telephone intervention showed a statistically significant effect in improved fatigue compared to placebo even if overall symptom burden was decreased [38]. Additionally, a recent placebo controlled RCT $(n=100)$ could not establish evidence for the use of methylphenidate $10 \mathrm{mg} /$ day for 6 days, even if fatigue was significantly improved in both groups [33].

\subsection{Corticosteroids}

Corticosteroids have in randomized trials showed to alleviate CRF. Patients with advanced colorectal cancer showed improved fatigue, receiving dexamethasone $2 \mathrm{mg} /$ day for four weeks during chemotherapy in a randomized placebo-controlled study [14]. In addition, dexamethasone $8 \mathrm{mg} /$ day for two weeks have also provided significant effect in alleviating fatigue in patients with advanced cancer $(n=84)$, in a randomized placebocontrolled trial [48]. In contrast, a randomized placebo-controlled study of patients with advanced cancer receiving methylprednisolone $32 \mathrm{mg} /$ day for 7 days also showed no effect [47]. Additionally, this study was powered for 110 patients but succeeded only to include 57 participants for the analysis. However, a significant effect in alleviating fatigue was established in a placebo-controlled RCT of patients with advanced cancer $(n=49)$ receiving methylprednisolone $32 \mathrm{mg}$ /day for one week [46].

\subsection{Melatonin}

Studies have been performed to test if improved sleep quality could alleviate cancer related fatigue. In a randomized placebo-controlled crossover study, patients with advanced cancer received melatonin $20 \mathrm{mg}$ /day or placebo for one week, which had no significant effect on improving fatigue [44]. 


\subsection{Testosterone}

Evidence for an effect in testosterone treatment in fatigue is yet not established due to few RCT's performed. A randomized placebo-controlled study of men with low testosterone and advanced cancer $(n=29)$ could not provide significant evidence for the use of testosterone 150-200 mg for alleviate fatigue after 29 days of treatment [45]. However, at day 72 , a significant effect was seen in the intervention arm in one of the subscale scores.

\section{Dietary Supplements and Complementary Medicine}

Different dietary supplements and pharmacological complementary and alternative medicines (CAMs) have been suggested to reduce CRF. However, there are only a few randomized studies performed in the palliative setting.

\subsection{Palliative Cancer Care Studies}

There are two randomized, placebo-controlled, double-blind studies performed in patients with advanced cancer, comparing L-carnitine with placebo [49,50]. Carnitine is an amino acid involved in the energy metabolism within the cell and it is suggested that a deficiency may contribute to fatigue. The first pilot study included 29 patients with advanced cancer and carnitine deficiency at baseline and the larger study included 376 patients with invasive malignancy. Both studies showed no effect of the intervention.

Ginseng has been used for several different conditions in the traditional Chinese medicine for thousands of years. Only one randomized, placebo-controlled, doubleblind study using American Ginseng (Panax ginseng) on the treatment of CRF has been performed in patients with advanced cancer $(n=112)$ [51]. Interestingly, an improvement of fatigue was shown in both treatment arms, but ginseng was not superior to the placebo.

It has been suggested that correction of vitamin D deficiency may have a positive effect on fatigue in palliative cancer patients [52-54]. This is currently investigated in a large ongoing randomized, placebo-controlled, double-blind study, "Palliative-D" [55]. The results from this study are still not published but in the baseline data from the study cohort $(n=530)$ there was a significant association between low vitamin $\mathrm{D}$ levels and severe fatigue in men but not in women [56].

\subsection{Non-Palliative Cancer Care Studies}

Except for the above-mentioned studies, the majority of studies on CAM have been performed in cancer patients in an earlier, non-palliative phase, of the disease. These studies include two randomized studies on the antioxidant Q10 that showed no or limited effect $[57,58]$ and four negative RCTs on the herbal drug Guarana [59-62]. In contrast, Tualang honey, suggested to have anti-inflammatory and antioxidative properties, was reported to have a significant positive effect on both fatigue and QoL compared to vitamin $\mathrm{C}$ treatment in a randomized, open-label study on head-and-neck cancer patients [63].

In contrast to the ginseng study in advanced cancer patients mentioned above, ginseng has shown promising effects in studies performed in cancer survivors and cancer patients in an earlier stage of the disease [64-66]. One of these studies was a large multicenter, double-blind RCT $(n=364)$ showing that American ginseng was superior compared to placebo after 8 weeks treatment [64]. All three studies report that the treatment was safe and well tolerated with no serious adverse events.

In a recent meta-analysis of traditional Chinese medicine (TCM) for treatment of CRF 17 randomized controlled trials of different herbal therapies were identified, of which the majority showed positive effects [67]. None of these studies were performed in a palliative care setting. The authors conclude that several of the herbal studies had poor methodological quality, with especially a lack of adequate randomization procedure and blinding.

\section{Discussion}

The result from this review showed that methylphenidate and corticosteroids may improve CRF in the palliative phase $[14,34,35,39,46,48]$ but there is not enough evidence 
to propose use of modafinil, dexamphetamine, melatonin or testosterone in this setting. It should be noted that the studied cohorts are heterogeneous and include patients both in the early and late palliative phase. Since fatigue is experienced differently throughout the disease trajectory and seems to affect QoL more in the early than in the late palliative phase [8], the comparison of results from these heterogeneous studies are problematic. Thus, the recommendations for different pharmacological agents to relieve fatigue are difficult.

The expert panel from NCCN focuses on patients during/after primary treatment and End-of-Life patients and does not specifically offer guidance on fatigue treatment in patients receiving active oncological treatment in a palliative setting [4]. Patients with metastatic disease receiving active oncological treatment may have a life expectancy of several years, and results from these cohorts cannot readily be compared with results from studies in patients with a life expectancy of a few weeks. Meta-analyses and group comparisons are therefore of little use at present, and instead we suggest that each study should be evaluated individually regarding generalizability of results. Unfortunately, characterization of disease severity in patients included in several studies in this review is scarce, and survival time is only accounted for in two trials $[14,33]$. Instead, inclusion and exclusion criteria, description of care facilities from which patients were recruited, assessed performance status at baseline and data on dropout rates and reasons for not completing intervention can be used to estimate general disease burden in studied cohorts. Additionally, this is discussed more in detail in the following sections.

\subsection{Psychostimulants (Methylphenidate, Dexamphetamine and Modafinile)}

Psychostimulants was the most commonly studied pharmacological interventions in studies on CRF in palliative care [33-43]. The results in this review revealed a significant effect on the primary outcome only in three trials out of eleven on methylphenidate and modafinil $[34,35,39]$. Several trials indicated partial, or a trend towards a positive effect during the time of intervention. Interestingly, several studies showed a significant effect in both treatment arms with similar effects in both placebo and intervention groups indicating an important placebo effect for any pharmacological treatment [33,36-43]. Most of the included studies were underpowered resulting in inconclusive data [33,35,37-39]. Regarding methylphenidate, earlier studies indicate that they can reverse sedating effects of opioids [68,69] and reduce depressive symptoms [70], both contributors to fatigue. However, a more recent study failed to see an association between effect of methylphenidate and daily opioid dose or depression [71].

Regarding RCTs on psychostimulants, we assessed that three studies were conducted on patients late in the disease trajectory: Centeno [33] — patients recruited from palliative care centers with short remaining life expectancy, Pedersen [34]—palliative inpatients with heavy symptom load and Mitchell [36] - patients who were not candidates for active oncological treatment recruited from palliative care facilities. Other trials mix palliative and curative patients recruited from oncology clinics [35,37], or include very little information on disease severity of randomized patients, rendering interpretation of results more difficult [38,39]. The trial on dexamphetamine [43] was also assessed as being performed in late stage palliative patients.

The three studies on modafinil all recruited patients from oncology centers in comparatively good performance status. Lee studied the effect of modafinil in patients with glioma with good physical performance status, starting high-dose radiotherapy and in some cases concomitant radiotherapy + chemotherapy [40]. Spathis et al. [41] recruited both possibly curative patients (lung cancer stage $3 \mathrm{a} / 3 \mathrm{~b}$ having completed first line treatment) and patients with metastatic and recurrent disease. Still, dropout due to deterioration or death was only $8 \%$, substantially lower than the expected $25-40 \%$ in more severely diseased palliative cohorts [41]. Hovey et al. recruited patients with ongoing docetaxel therapy, with no dropout due to deterioration or death during the intervention period, indication a cohort in early palliative phase [42]. 
Psychostimulants in low doses, as used in the palliative care setting, are often welltolerated and have no or only mild side-effects reported as evenly distributed between treatment and placebo arm [33,35-41]. There seems to be responders and non-responders to this treatment $[36,43]$ —and thus it might be suggested to test this treatment option despite the limited evidence from the underpowered studies. The effect has a rapid onset and is often evident already after the first dose. Thus, the effect could be evaluated already after a few days and terminated if there is a lack of effect or continued if the effect is positive. Methylphenidate might be considered as a first choice since it is the only psychostimulant that has shown a superior effect over placebo in three RCTs $[34,35,39]$ while all RCTs on modafinil have been negative [40-42].

\subsection{Corticosteroids}

In clinical practice, corticosteroids are widely used in cancer patients in the palliative phase to alleviate inflammatory pain, relieve nausea, reduce peritumoral oedema, increase appetite, reduce itching and to reduce fatigue [72,73]. As shown above, clinical trials investigating the effect of corticosteroids on fatigue in patients with advanced or metastatic cancer are scarce and partly based on observational studies [74,75]. Of the four RCTs included in this review one showed negative results [47]. Thus, treatment decisions are based rather on empirical knowledge rather scientific evidence, and neither optimal dose nor length of treatment has been established. Side effects of corticosteroids in this setting have not been systematically assessed in clinical trials $[74,76]$. Still, NCCN for the first time now include short courses of corticosteroids as a treatment options for End-of-Life patients [4]. Further trials on the use of corticosteroids in to relieve fatigue in palliative care are warranted. In their review from 2014, Yennurajalingam et al. argue that trials to study pharmacological interventions in fatigue should be tailored to recruit patients that are most likely to experience a beneficial effect of the studied compound [1]. In the case of steroids, they proposed to study the effect in patients with evident inflammation, measured with CRP, cytokines or other agents [1]. Other ideas would be to study the effect and side effects of corticosteroids on fatigue in patients with liver metastases irrespective of CRP-levels, slightly longer treatment periods with lower doses, and the effect of repeated shorter courses (as n-of-1-trials). Regarding studies on corticosteroids, three out of four included trials are assessed as including patients in the later phases of their disease trajectories [46-48], whilst the study by Tanioka [14] included patients with ongoing active treatment and overall survival of 6.8 months.

\subsection{Melatonin}

The RCT on melatonine [44] had relatively large dropout rates due to deterioration or death, and thus included many end-stage cancer patients. Interestingly, melatonin has, in addition to its effect on sleep disorder, been suggested to have anti-inflammatory and antioxidative effects on a cellular level [77]. Thus, we believe that melatonin is an interesting agent for treatment of fatigue and deserves further studies.

\subsection{Testosterone}

The Del Fabbros trial on testosterone involved oncological outpatients with metastatic or recurrent cancers, with no dropout during follow-up, indicating that this cohort had a comparatively long life expectancy [45].

\subsection{Complementary and Alternative Medicine}

A recent review suggests the need of addressing CAM in treating CRF since patients may use this as part of self-care, alongside with traditional treatment methods without informing health care providers [78]. Except for three high-quality studies performed in patients with advanced cancer, including interventions with L-carnitin and ginseng [49-51], no studies have been performed in the palliative setting. All three studies showed negative results. Indeed, most pharmacological CAM interventions on cancer patients in 
an earlier phase of the disease are also negative or are have been performed with low methodological quality. An exception might be the high-quality study performed on ginseng that shows promising effects on CRF in cancer survivors [64]. A review of the use of guarana, ginseng, acetyl L-carnitine, Q10 enzyme and green algae demonstrates that trials are mostly performed with women with breast cancer in all stages and does not specifically target palliative care [79]. Further research of CAM in palliative context is needed to provide evidence.

\subsection{Placebo Effect}

Interestingly, several of the studies described in this review show an improvement of fatigue both in the intervention and the placebo arm where the intervention is not superior to placebo. Two recent reviews have highlighted that placebo may affect fatigue in patients with cancer $[80,81]$, which is an important confounding factor when analysis of intervention is performed. The authors to one of these reviews recommend that it might be necessary to develop alternative trial designs to better account for the placebo response when studying effect on fatigue [81]. A study of placebo responses on CRF confirmed that up to $29 \%$ of patients with advanced cancer experienced improved fatigue compared to usual care [82], indicating that the mechanism behind responses are complex. However, in the clinical setting also the placebo effect is an important effect. If the treatment has no adverse side effects any positive effect of the treatment is beneficial for the patient.

\subsection{Mixed Interventions}

There is a lack of trials using methods of both pharmacological and non-pharmacological methods to alleviate fatigue. However, studies performed with mixed interventions have also failed to show a significant effect in alleviating fatigue [38]. NCCN recommends primarily non-pharmacological approaches, such as physical activity, counseling and nutritional support to treat CRF after treatment and in End-of-Life care. This is supported in a recent review comparing pharmacological and non-pharmacological interventions on fatigue in patients with cancer during and after treatment [10].

\subsection{Clinical Applications}

According to the findings presented here corticosteroids and methylphenidate might be the drugs of choice for pharmacological treatment of fatigue in palliative cancer care. However, the placebo effect is not negligible for any of the treatments studied. In addition, the patients included in the studied presented here is heterogeneous and patients in the very End-of-Life is mostly missing in clinical trials. Thus, when the optimal treatment for an individual patient is decided, several factors have to be taken into account, such as the performance status of the patient, the motivation and belief in the suggested treatment, and which adverse reactions that should be avoided to sustain the QoL of this specific patient. Above all, the treatment should not harm the patient in any way. Thus, we recommend an individualized treatment approach rather than a generalized recommendation that fits all.

\section{Limitations and Strengths}

This review had several limitations. First, this is a narrative review and no metaanalysis was performed. Second, only RCT trials were included, targeting the palliative phase, and there may be some trials left out, which may result in a lack of efficiency detected in other pharmacological interventions or with different design. Third, trials combining non-pharmacological and pharmacological interventions have not been included. Additionally, the variety of assessment tools makes it difficult to identify the impact of coexisting factors affecting the effect on CRF such as the psychological, physical and emotional status and depression. A strength of this review is the focus on CRF and pharmacological interventions in cancer disease in the palliative phase, which may differ from the pharmacological effects in curable disease. 


\section{Conclusions}

To conclude, the evidence is still weak for using pharmacological treatments on fatigue in palliative care patients, although methylphenidate and corticosteroids might be considered. Further research is needed regarding pharmacological treatments on CRF in patients with advanced cancer admitted to palliative care, and in combination with non-pharmacological interventions, and about CRF in End-of-Life. Additionally, further research of biological mechanisms affecting fatigue is needed to support and raise new research questions and interventions.

Author Contributions: Conceptualization, C.K., C.L.H. and L.B.-B.; methodology, C.K., M.H.F., C.L.H., L.B.-B.; validation, C.K., C.L.H., L.B.-B.; writing—original draft preparation, C.K.; writingreview and editing, C.K., M.H.F., C.L.H., L.B.-B.; visualization, M.H.F.; supervision, C.L.H., L.B.B.; funding acquisition, L.B.-B. All authors have read and agreed to the published version of the manuscript.

Funding: This study was financially supported by grants from Stockholm County Council (SLL20160036 and SLL20180320), the Swedish Cancer Society (CAN 2017/233 and CAN2018/316), Stockholms Sjukhems Jubileumsfond.

Conflicts of Interest: No financial interests have been declared by the authors.

\section{References}

1. Yennurajalingam, S.; Bruera, E. Review of Clinical Trials of Pharmacologic Interventions for Cancer-Related Fatigue. Cancer J. 2014, 20, 319-324. [CrossRef] [PubMed]

2. Radbruch, L.; Strasser, F.; Elsner, F.; Gonçalves, J.F.; Løge, J.; Kaasa, S.; Nauck, F.; Stone, P. Fatigue in palliative care patients-An EAPC approach. Palliat. Med. 2008, 22, 13-32. [CrossRef] [PubMed]

3. Bower, J.E. Cancer-related fatigue-Mechanisms, risk factors, and treatments. Nat. Rev. Clin. Oncol. 2014, 11, 597-609. [CrossRef]

4. National Comprehensive Cancer Network. NCCN Guidelines Version 1.20201 Cancer-Related Fatigue. 2020. Available online: nccn.org (accessed on 15 December 2020).

5. Thong, M.S.Y.; Mols, F.; Van De Poll-Franse, L.V.; Sprangers, M.A.G.; Van Der Rijt, C.C.D.; Barsevick, A.M.; Knoop, H.; Husson, O. Identifying the subtypes of cancer-related fatigue: Results from the population-based PROFILES registry. J. Cancer Surviv. 2017, 12, 38-46. [CrossRef] [PubMed]

6. De Raaf, P.J.; De Klerk, C.; Van Der Rijt, C.C.D. Elucidating the behavior of physical fatigue and mental fatigue in cancer patients: A review of the literature. Psychooncology 2012, 22, 1919-1929. [CrossRef] [PubMed]

7. Hagelin, C.L.; Wengström, Y.; Fürst, C.J. Patterns of fatigue related to advanced disease and radiotherapy in patients with cancer-A comparative cross-sectional study of fatigue intensity and characteristics. Support. Care Cancer 2008, 17, 519-526. [CrossRef]

8. Hagelin, C.L.; Wengstrom, Y.; Ahsberg, E.; Furst, C.J. Fatigue dimensions in patients with advanced cancer in relation to time of survival and quality of life. Palliat. Med. 2009, 23, 171-178. [CrossRef]

9. De Raaf, P.J.; De Klerk, C.; Timman, R.; Hinz, A.; Van Der Rijt, C.C. Differences in Fatigue Experiences Among Patients with Advanced Cancer, Cancer Survivors, and the General Population. J. Pain Symptom Manag. 2012, 44, 823-830. [CrossRef]

10. Mustian, K.M.; Alfano, C.M.; Heckler, C.; Kleckner, A.S.; Kleckner, I.R.; Leach, C.R.; Mohr, D.; Palesh, O.G.; Peppone, L.J.; Piper, B.F.; et al. Comparison of Pharmaceutical, Psychological, and Exercise Treatments for Cancer-Related Fatigue. JAMA Oncol. 2017, 3, 961-968. [CrossRef]

11. Al Maqbali, M.; Hughes, C.; Gracey, J.; Rankin, J.; Dunwoody, L.; Hacker, E. Quality assessment criteria: Psychometric properties of measurement tools for cancer related fatigue. Acta Oncol. 2019, 58, 1286-1297. [CrossRef] [PubMed]

12. Al Maqbali, M.; Al Sinani, M.; Al Naamani, Z.; Al Badi, K.; Tanash, M.I. Prevalence of Fatigue in Patients with Cancer: A Systematic Review and Meta-Analysis. J. Pain Symptom Manag. 2021, 61, 167-189. [CrossRef]

13. Mock, V. Fatigue management: Evidence and guidelines for practice. Cancer 2001, 92, 1699-1707. [CrossRef]

14. Tanioka, H.; Miyamoto, Y.; Tsuji, A.; Asayama, M.; Shiraishi, T.; Yuki, S.; Kotaka, M.; Makiyama, A.; Shimokawa, M.; Shimose, T.; et al. Prophylactic Effect of Dexamethasone on Regorafenib-Related Fatigue and/or Malaise: A Randomized, Placebo-Controlled, Double-Blind Clinical Study in Patients with Unresectable Metastatic Colorectal Cancer (KSCC1402/HGCSG1402). Oncology 2018, 94, 289-296. [CrossRef] [PubMed]

15. WHO. Definition of Palliative Care. Available online: http://www.who.int/cancer/palliative/definition/en/ (accessed on 5 October 2020).

16. Verkissen, M.N.; Hjermstad, M.J.; Van Belle, S.; Kaasa, S.; Deliens, L.; Pardon, K. Quality of life and symptom intensity over time in people with cancer receiving palliative care: Results from the international European Palliative Care Cancer Symptom study. PLOS ONE 2019, 14, e0222988. [CrossRef] [PubMed] 
17. Radbruch, L.; De Lima, L.; Knaul, F.; Wenk, R.; Ali, Z.; Bhatnaghar, S.; Blanchard, C.; Bruera, E.; Buitrago, R.; Burla, C.; et al. Redefining Palliative Care-A New Consensus-Based Definition. J. Pain Symptom Manag. 2020, 60, 754-764. [CrossRef]

18. Poort, H.; Peters, M.; Bleijenberg, G.; Gielissen, M.F.; Goedendorp, M.M.; Jacobsen, P.; Verhagen, S.; Knoop, H. Psychosocial interventions for fatigue during cancer treatment with palliative intent. Cochrane Database Syst. Rev. 2017, 2017. [CrossRef] [PubMed]

19. Mücke, M.; Mochamat, M.; Cuhls, H.; Peuckmann-Post, V.; Minton, O.; Stone, P.; Radbruch, L. Pharmacological treatments for fatigue associated with palliative care. Cochrane Database Syst. Rev. 2015, 2015, CD006788. [CrossRef]

20. Tomlinson, D.; Robinson, P.D.; Oberoi, S.; Cataudella, D.; Culos-Reed, N.; Davis, H.; Duong, N.; Gibson, F.; Götte, M.; Hinds, P.; et al. Pharmacologic Interventions for Fatigue in Cancer and Transplantation: A Meta-Analysis. Curr. Oncol. 2018, 25, 152-167. [CrossRef] [PubMed]

21. Qu, D.; Zhang, Z.; Yu, X.; Zhao, J.; Qiu, F.; Huang, J. Psychotropic drugs for the management of cancer-related fatigue: A systematic review and meta-analysis. Eur. J. Cancer Care 2016, 25, 970-979. [CrossRef]

22. Begley, S.; Rose, K.; O'Connor, M. The use of corticosteroids in reducing cancer-related fatigue: Assessing the evidence for clinical practice. Int. J. Palliat. Nurs. 2016, 22, 5-9. [CrossRef] [PubMed]

23. Bower, J.E.; Bak, K.; Berger, A.; Breitbart, W.; Escalante, C.P.; Ganz, P.A.; Schnipper, H.H.; Lacchetti, C.; Ligibel, J.A.; Lyman, G.H.; et al. Screening, Assessment, and Management of Fatigue in Adult Survivors of Cancer: An American Society of Clinical Oncology Clinical Practice Guideline Adaptation. J. Clin. Oncol. 2014, 32, 1840-1850. [CrossRef]

24. Koornstra, R.H.; Peters, M.; Donofrio, S.; Borne, B.V.D.; De Jong, F.A. Management of fatigue in patients with cancer-A practical overview. Cancer Treat. Rev. 2014, 40, 791-799. [CrossRef]

25. Ma, Y.; He, B.; Jiang, M.; Yang, Y.; Wang, C.; Huang, C.; Han, L. Prevalence and risk factors of cancer-related fatigue: A systematic review and meta-analysis. Int. J. Nurs. Stud. 2020, 111, 103707. [CrossRef]

26. Ingham, G.; Urban, K.; Allingham, S.F.; Blanchard, M.; Marston, C.; Currow, D.C. The Level of Distress from Fatigue Re-ported in the Final Two Months of Life by a Palliative Care Population: An Australian National Prospective, Consecutive Case Series. J. Pain Symptom Manag. 2020. [CrossRef] [PubMed]

27. Peters, M.E.; Goedendorp, M.M.; Verhagen, S.A.; Smilde, T.J.; Bleijenberg, G.; Van Der Graaf, W.T.A. A prospective analysis on fatigue and experienced burden in informal caregivers of cancer patients during cancer treatment in the palliative phase. Acta Oncol. 2015, 54, 500-506. [CrossRef]

28. Yang, S.; Chu, S.; Gao, Y.; Ai, Q.; Liu, Y.; Li, X.; Chen, N. A Narrative Review of Cancer-Related Fatigue (CRF) and Its Possible Pathogenesis. Cells 2019, 8, 738. [CrossRef] [PubMed]

29. O'Higgins, C.M.; Brady, B.; O'Connor, B.; Walsh, D.; Reilly, R.B. The pathophysiology of cancer-related fatigue: Current controversies. Support. Care Cancer 2018, 26, 3353-3364. [CrossRef]

30. Paulsen, Ø.; Laird, B.; Aass, N.; Lea, T.; Fayers, P.; Kaasa, S.; Klepstad, P. The relationship between pro-inflammatory cytokines and pain, appetite and fatigue in patients with advanced cancer. PLoS ONE 2017, 12, e0177620. [CrossRef]

31. Fabi, A.; Bhargava, R.; Fatigoni, S.; Guglielmo, M.; Horneber, M.; Roila, F.; Weis, J.; Jordan, K.; Ripamonti, C. Cancer-related fatigue: ESMO Clinical Practice Guidelines for diagnosis and treatment. Ann. Oncol. 2020, 31, 713-723. [CrossRef]

32. Vilchynska, T.; Beard, B. Cancer-related fatigue in palliative care: A global perspective. Int. J. Palliat. Nurs. 2016, $22,244-252$. [CrossRef]

33. Centeno, C.; Rojí, R.; Portela, M.A.; De Santiago, A.; Cuervo, M.A.; Ramos, D.; Gandara, A.; Salgado, E.; Gagnon, B.; Sanz, A. Improved cancer-related fatigue in a randomised clinical trial: Methylphenidate no better than placebo. BMJ Support. Palliat. Care 2020. [CrossRef]

34. Pedersen, L.; Lund, L.; Petersen, M.A.; Sjogren, P.; Groenvold, M. Methylphenidate as Needed for Fatigue in Patients with Advanced Cancer. A Prospective, Double-Blind, and Placebo-Controlled Study. J. Pain Symptom Manag. 2020, 60, 992-1002. [CrossRef]

35. Richard, P.O.; Fleshner, N.E.; Bhatt, J.R.; Hersey, K.M.; Chahin, R.; Alibhai, S.M. A Phase II, Randomized, Double-blind, PlaceboControlled Trial of Methylphenidate for Reduction of Fatigue in Prostate Cancer Patients Receiving LHRH-Agonist Therapy. BJU Int. 2015, 116, 744-752. [CrossRef]

36. Mitchell, G.K.; Hardy, J.R.; Nikles, C.J.; Carmont, S.-A.S.; Senior, H.E.; Schluter, P.J.; Good, P.; Currow, D.C. The Effect of Methylphenidate on Fatigue in Advanced Cancer: An Aggregated N-of-1 Trial. J. Pain Symptom Manag. 2015, 50, $289-296$. [CrossRef]

37. Escalante, C.P.; Meyers, C.; Reuben, J.M.; Wang, X.; Qiao, W.; Manzullo, E.; Alvarez, R.H.; Morrow, P.K.; Gonzalez-Angulo, A.M.; Wang, X.S.; et al. A Randomized, Double-blind, 2-Period, Placebo-Controlled Crossover Trial of a Sustained-Release Methylphenidate in the Treatment of Fatigue in Cancer Patients. Cancer J. 2014, 20, 8-14. [CrossRef]

38. Bruera, E.; Yennurajalingam, S.; Palmer, J.L.; Perez-Cruz, P.E.; Frisbee-Hume, S.; Allo, J.A.; Williams, J.L.; Cohen, M.Z. Methylphenidate and/or a Nursing Telephone Intervention for Fatigue in Patients with Advanced Cancer: A Randomized, Placebo-Controlled, Phase II Trial. J. Clin. Oncol. 2013, 31, 2421-2427. [CrossRef]

39. Roth, A.J.; Nelson, C.; Rosenfeld, B.; Scher, H.; Slovin, S.; Morris, M.; O'Shea, N.; Rn, G.A.; Breitbart, W. Methylphenidate for fatigue in ambulatory men with prostate cancer. Cancer 2010, 116, 5102-5110. [CrossRef] 
40. Lee, E.Q.; Muzikansky, A.; Drappatz, J.; Kesari, S.; Wong, E.T.; Fadul, C.E.; Reardon, D.A.; Norden, A.D.; Nayak, L.; Rinne, M.L.; et al. A randomized, placebo-controlled pilot trial of armodafinil for fatigue in patients with gliomas undergoing radiotherapy. Neurooncology 2016, 18, 849-854. [CrossRef]

41. Spathis, A.; Fife, K.; Blackhall, F.; Dutton, S.; Bahadori, R.; Wharton, R.; O’Brien, M.; Stone, P.; Benepal, T.; Bates, N.; et al. Modafinil for the Treatment of Fatigue in Lung Cancer: Results of a Placebo-Controlled, Double-Blind, Randomized Trial. J. Clin. Oncol. 2014, 32, 1882-1888. [CrossRef]

42. Hovey, E.; De Souza, P.; Marx, G.; Parente, P.; Rapke, T.; Hill, A.; Bonaventura, A.; Michele, A.; Craft, P.; On Behalf of the MOTIF Investigators; et al. Phase III, randomized, double-blind, placebo-controlled study of modafinil for fatigue in patients treated with docetaxel-based chemotherapy. Support. Care Cancer 2013, 22, 1233-1242. [CrossRef]

43. Auret, K.A.; Schug, S.A.; Bremner, A.P.; Bulsara, M. A Randomized, Double-Blind, Placebo-Controlled Trial Assessing the Impact of Dexamphetamine on Fatigue in Patients with Advanced Cancer. J. Pain Symptom Manag. 2009, 37, 613-621. [CrossRef] [PubMed]

44. Rasmussen, C.L.; Olsen, M.K.; Johnsen, A.T.; Petersen, M.A.; Lindholm, H.; Andersen, L.; Villadsen, B.; Groenvold, M.; Pedersen, L. Effects of melatonin on physical fatigue and other symptoms in patients with advanced cancer receiving palliative care: A double-blind placebo-controlled crossover trial. Cancer 2015, 121, 3727-3736. [CrossRef]

45. Del Fabbro, E.; Garcia, J.M.; Dev, R.; Hui, D.; Williams, J.; Engineer, D.; Palmer, J.L.; Schover, L.; Bruera, E. Testosterone replacement for fatigue in hypogonadal ambulatory males with advanced cancer: A preliminary double-blind placebo-controlled trial. Support. Care Cancer 2013, 21, 2599-2607. [CrossRef]

46. Paulsen, Ø.; Klepstad, P.; Rosland, J.H.; Aass, N.; Albert, E.; Fayers, P.; Kaasa, S. Efficacy of Methylprednisolone on Pain, Fatigue, and Appetite Loss in Patients with Advanced Cancer Using Opioids: A Randomized, Placebo-Controlled, Double-Blind Trial. J. Clin. Oncol. 2014, 32, 3221-3228. [CrossRef] [PubMed]

47. Eguchi, K.; Honda, M.; Kataoka, T.; Mukouyama, T.; Tsuneto, S.; Sakamoto, J.; Oba, K.; Saji, S. Efficacy of corticosteroids for cancer-related fatigue: A pilot randomized placebo-controlled trial of advanced cancer patients. Palliat. Support. Care 2014, 13, 1301-1308. [CrossRef]

48. Yennurajalingam, S.; Frisbee-Hume, S.; Palmer, J.L.; Delgado-Guay, M.O.; Bull, J.; Phan, A.T.; Tannir, N.M.; Litton, J.K.; Reddy, A.; Hui, D.; et al. Reduction of Cancer-Related Fatigue with Dexamethasone: A Double-Blind, Randomized, Placebo-Controlled Trial in Patients with Advanced Cancer. J. Clin. Oncol. 2013, 31, 3076-3082. [CrossRef]

49. Cruciani, R.A.; Dvorkin, E.; Homel, P.; Culliney, B.; Malamud, S.; Lapin, J.; Portenoy, R.K.; Esteban-Cruciani, N. L-Carnitine Supplementation in Patients with Advanced Cancer and Carnitine Deficiency: A Double-Blind, Placebo-Controlled Study. J. Pain Symptom Manag. 2009, 37, 622-631. [CrossRef]

50. Cruciani, R.A.; Zhang, J.J.; Manola, J.; Cella, D.; Ansari, B.; Fisch, M.J. L-Carnitine Supplementation for the Management of Fatigue in Patients with Cancer: An Eastern Cooperative Oncology Group Phase III, Randomized, Double-Blind, Placebo-Controlled Trial. J. Clin. Oncol. 2012, 30, 3864-3869. [CrossRef]

51. Yennurajalingam, S.; Tannir, N.M.; Williams, J.L.; Lu, Z.; Hess, K.R.; Frisbee-Hume, S.; House, H.L.; Lim, Z.D.; Lim, K.-H.; Lopez, G.; et al. A Double-Blind, Randomized, Placebo-Controlled Trial ofPanax Ginsengfor Cancer-Related Fatigue in Patients with Advanced Cancer. J. Natl. Compr. Cancer Netw. 2017, 15, 1111-1120. [CrossRef]

52. Björkhem-Bergman, L.; Bergman, P. Vitamin D and patients with palliative cancer. BMJ Support. Palliat. Care 2016, 6, 287-291. [CrossRef]

53. Helde-Frankling, M.; Björkhem-Bergman, L. Vitamin D in Pain Management. Int. J. Mol. Sci. 2017, 18, 2170. [CrossRef]

54. Koole, J.L.; Bours, M.J.; Van Roekel, E.H.; Breedveld-Peters, J.J.; Van Duijnhoven, F.J.; Ouweland, J.V.D.; Breukink, S.O.; JanssenHeijnen, M.L.; Keulen, E.T.; Weijenberg, M.P. Higher Serum Vitamin D Concentrations Are Longitudinally Associated with Better Global Quality of Life and Less Fatigue in Colorectal Cancer Survivors up to 2 Years after Treatment. Cancer Epidemiol. Biomark. Prev. 2020, 29, 1135-1144. [CrossRef]

55. Helde-Frankling, M.; Bergqvist, J.; Klasson, C.; Nordström, M.; Höijer, J.; Bergman, P.; Björkhem-Bergman, L. Vitamin D supplementation to palliative cancer patients: Protocol of a double-blind, randomised controlled trial 'Palliative-D'. BMJ Support. Palliat. Care 2017, 7, 458-463. [CrossRef]

56. Klasson, C.; Helde-Frankling, M.; Sandberg, C.; Nordström, M.; Lundh-Hagelin, C.; Björkhem-Bergman, L. Vitamin D and Fatigue in Palliative Cancer: A Cross-Sectional Study of Sex Difference in Baseline Data from the Palliative D Cohort. J. Palliat. Med. 2020. [CrossRef]

57. Iwase, S.; Kawaguchi, T.; Yotsumoto, D.; Doi, T.; Miyara, K.; Odagiri, H.; Kitamura, K.; Ariyoshi, K.; Miyaji, T.; Ishiki, H.; et al. Efficacy and safety of an amino acid jelly containing coenzyme Q10 and l-carnitine in controlling fatigue in breast cancer patients receiving chemotherapy: A multi-institutional, randomized, exploratory trial (JORTC-CAM01). Support. Care Cancer 2015, 24, 637-646. [CrossRef]

58. Lesser, G.J.; Case, U.; Stark, N.; Williford, S.; Giguere, J.; Garino, L.A.; Naughton, M.J.; Vitolins, M.Z.; Lively, M.O.; Shaw, E.G.; et al. A randomized, double-blind, placebo-controlled study of oral coenzyme Q10 to relieve self-reported treatment-related fatigue in newly diagnosed patients with breast cancer. J. Support. Oncol. 2012, 11, 31-42. [CrossRef]

59. Miranda, V.D.C.; Trufelli, D.C.; Santos, J.; Campos, M.P.; Nobuo, M.; Miranda, M.D.C.; Schlinder, F.; Riechelmann, R.; Del Giglio, A. Effectiveness of Guaraná (Paullinia cupana) for Postradiation Fatigue and Depression: Results of a Pilot Double-Blind Randomized Study. J. Altern. Complement. Med. 2009, 15, 431-433. [CrossRef] 
60. Del Giglio, A.B.; Cubero, D.D.I.G.; Lerner, T.G.; Guariento, R.T.; De Azevedo, R.G.S.; Paiva, H.; Goldman, C.; Carelli, B.; Cruz, F.M.; Schindler, F.; et al. Purified Dry Extract ofPaullinia cupana(Guaraná) (PC-18) for Chemotherapy-Related Fatigue in Patients with Solid Tumors: An Early Discontinuation Study. J. Diet. Suppl. 2013, 10, 325-334. [CrossRef]

61. Martins, S.P.D.S.; Ferreira, C.L.; Del Giglio, A. Placebo-Controlled, Double-Blind, Randomized Study of a Dry Guarana Extract in Patients with Head and Neck Tumors Undergoing Chemoradiotherapy: Effects on Fatigue and Quality of Life. J. Diet. Suppl. 2016, 14, 32-41. [CrossRef]

62. Sette, C.V.D.M.; De Alcântara, B.B.R.; Schoueri, J.H.M.; Cruz, F.M.; Cubero, D.D.I.G.; Pianowski, L.F.; Peppone, L.J.; Fonseca, F.; Del Giglio, A. Purified Dry Paullinia cupana (PC-18) Extract for Chemotherapy-Induced Fatigue: Results of Two Double-Blind Randomized Clinical Trials. J. Diet. Suppl. 2017, 15, 673-683. [CrossRef]

63. Ramasamy, V.; Binti Mat Lazim, N.; Abdullah, B.; Singh, A. Effects of Tualang Honey on Cancer Related Fatigue: A Multi-center Open-label Trial of H\&N Cancer Patients. Gulf J. Oncol. 2019, 1, 43-51.

64. Barton, D.L.; Liu, H.; Dakhil, S.R.; Linquist, B.; Sloan, J.A.; Nichols, C.R.; McGinn, T.W.; Stella, P.J.; Seeger, G.R.; Sood, A.; et al. Wisconsin Ginseng (Panax quinquefolius) to Improve Cancer-Related Fatigue: A Randomized, Double-Blind Trial, N07C2. J. Natl. Cancer Inst. 2013, 105, 1230-1238. [CrossRef]

65. Barton, D.L.; Soori, G.S.; Bauer, B.A.; Sloan, J.A.; Johnson, P.A.; Figueras, C.; Duane, S.; Mattar, B.; Liu, H.; Atherton, P.J.; et al. Pilot study of Panax quinquefolius (American ginseng) to improve cancer-related fatigue: A randomized, double-blind, dose-finding evaluation: NCCTG trial N03CA. Support. Care Cancer 2010, 18, 179-187. [CrossRef]

66. Jeong, J.S.; Ryu, B.H.; Kim, J.S.; Park, J.W.; Choi, W.C.; Yoon, S.W. Bojungikki-Tang for Cancer-Related Fatigue: A Pilot Randomized Clinical Trial. Integr. Cancer Ther. 2010, 9, 331-338. [CrossRef]

67. Zhao, Y.; Wang, S.; Li, J.; Zhou, Y.; Wu, W.; Sunny, H.S. Effectiveness and safety of traditional Chinese medical therapy for cancer-related fatigue: A systematic review and Meta-analysis of randomized controlled trials. J. Tradit. Chin. Med. 2020, 40, 738-748.

68. Bruera, E.; Kuehn, N.; Miller, M.J.; Selmser, P.; Macmillan, K. The Edmonton Symptom Assessment System (ESAS): A Simple Method for the Assessment of Palliative Care Patients. J. Palliat. Care 1991, 7, 6-9. [CrossRef]

69. Bruera, E.; Miller, M.J.; Macmillan, K.; Kuehn, N. Neuropsychological effects of methylphenidate in patients receiving a continuous infusion of narcotics for cancer pain. Pain 1992, 48, 163-166. [CrossRef]

70. Homsi, J.; Nelson, K.A.; Sarhill, N.; Rybicki, L.; Legrand, S.B.; Davis, M.P.; Walsh, D. A phase II study of methylphenidate for depression in advanced cancer. Am. J. Hosp. Palliat. Med. 2001, 18, 403-407. [CrossRef]

71. Yennurajalingam, S.; Palmer, J.L.; Chacko, R.; Bruera, E. Factors Associated with Response to Methylphenidate in Advanced Cancer Patients. Oncology 2011, 16, 246-253. [CrossRef]

72. Hardy, J.; Haywood, A.; Rickett, K.; Sallnow, L.; Good, P. Practice review: Evidence-based quality use of corticosteroids in the palliative care of patients with advanced cancer. Palliat. Med. 2021. [CrossRef]

73. Jaward, L.R.; O'Neil, T.A.; Marks, A.; Smith, M.A. Differences in Adverse Effect Profiles of Corticosteroids in Palliative Care Patients. Am. J. Hosp. Palliat. Med. 2019, 36, 158-168. [CrossRef]

74. Pinkerton, E.; Good, P.; Kindl, K.; Richard, R.; Fischer, A.; Hardy, J.R. Quality use of medicines: Oral corticosteroids in advanced cancer. Palliat. Med. 2019, 33, 1325-1326. [CrossRef]

75. Hardy, J.R.; Rees, E.; Ling, J.; Burman, R.; Feuer, D.; Broadley, K.; Stone, P. A prospective survey of the use of dexamethasone on a palliative care unit. Palliat. Med. 2001, 15, 3-8. [CrossRef]

76. Hatano, Y.; Matsuoka, H.; Lam, L.; Currow, D.C. Side effects of corticosteroids in patients with advanced cancer: A systematic review. Support. Care Cancer 2018, 26, 3979-3983. [CrossRef]

77. Favero, G.; Franceschetti, L.; Bonomini, F.; Rodella, L.F.; Rezzani, R. Melatonin as an Anti-Inflammatory Agent Modulating Inflammasome Activation. Int. J. Endocrinol. 2017, 2017, 1-13. [CrossRef]

78. Thong, M.S.; Van Noorden, C.J.F.; Steindorf, K.; Arndt, V. Cancer-Related Fatigue: Causes and Current Treatment Options. Curr. Treat. Opt. Oncol. 2020, 21, 1-19. [CrossRef] [PubMed]

79. Pereira, P.T.V.T.; Reis, A.D.; Diniz, R.R.; Lima, F.A.; Leite, R.D.; Da Silva, M.C.P.; Guerra, R.N.M.; de Moraes Vieira, É.B.; Garcia, J.B.S. Dietary supplements and fatigue in patients with breast cancer: A systematic review. Breast Cancer Res. Treat. 2018, 171, 515-526. [CrossRef]

80. Junior, P.N.A.; Barreto, C.M.N.; Cubero, D.D.I.G.; Del Giglio, A. The efficacy of placebo for the treatment of cancer-related fatigue: A systematic review and meta-analysis. Support. Care Cancer 2019, 28, 1755-1764. [CrossRef]

81. Roji, R.; Stone, P.; Ricciardi, F.; Candy, B. Placebo response in trials of drug treatments for cancer-related fatigue: A systematic review, meta-analysis and meta-regression. BMJ Support. Palliat. Care 2020, 10, 385-394. [CrossRef] [PubMed]

82. Hoenemeyer, T.W.; Kaptchuk, T.J.; Mehta, T.S.; Fontaine, K.R. Open-Label Placebo Treatment for Cancer-Related Fatigue: A Randomized-Controlled Clinical Trial. Sci. Rep. 2018, 8, 1-8. [CrossRef] [PubMed] 